\title{
Lithography-based additive manufacture of ceramic biodevices with design-controlled surface topographies
}

\author{
Adrián de Blas Romero ${ }^{1}$ • Markus Pfaffinger ${ }^{2}$. Gerald Mitteramskogler ${ }^{3}$. \\ Martin Schwentenwein ${ }^{3}$ - Christopher Jellinek ${ }^{3}$ • Johannes Homa ${ }^{3}$ • \\ Andrés Díaz Lantada ${ }^{1}$ • Jürgen Stampfl ${ }^{2,4}$
}

Received: 21 December 2015 / Accepted: 26 April 2016/Published online: 16 May 2016

(C) The Author(s) 2016. This article is published with open access at Springerlink.com

\begin{abstract}
The possibility of manufacturing textured materials and devices, with surface properties controlled from the design stage, instead of being the result of machining processes or chemical attacks, is a key factor for the incorporation of advanced functionalities to a wide set of micro- and nanosystems. High-precision additive manufacturing (AM) technologies based on photopolymerization, together with the use of fractal models linked to computer-aided design tools, allow for a precise definition of final surface properties. However, the polymeric master parts obtained with most commercial systems are usually inadequate for biomedical purposes and their limited strength and size prevents many potential applications. On the other hand, additive manufacturing technologies aimed at the production of final parts, normally based on layer-by-layer melting or sintering ceramic or metallic powders, do not always provide the required precision for obtaining controlled micro-structured surfaces with high-aspect-ratio details. Towards the desired degree of precision and performance, lithography-based ceramic manufacture is a remarkable option, as we discuss in the present study, which presents the development of two different micro-textured biodevices for cell culture. Results show a
\end{abstract}

Andrés Díaz Lantada

adiaz@etsii.upm.es

1 UPM Product Development Lab, Mechanical Engineering Department, Universidad Politécnica de Madrid (TU Madrid, www.upm.es), c/ José Gutiérrez Abascal 2, 28006 Madrid, Spain

2 Christian Doppler Laboratory for Digital and Restorative Dentistry, Vienna, Austria

3 Lithoz GmbH, Mollardgasse 85a/2/64-69, 1060 Vienna, Austria

4 Institute of Materials Science and Technology, TU Wien, Wien, Austria remarkable control of the surface topography of ceramic parts and the possibility of obtaining design-controlled micro-structured surfaces with high-aspect-ratio micro-metric details.

Keywords Fractals $\cdot$ Surface topography $\cdot$ Material texture · Materials design $\cdot$ Computer-aided design $\cdot$ Additive manufacturing $\cdot$ Lithography-based ceramic manufacture

\section{Introduction}

Material (and device) surface topography has a direct influence on several relevant properties, linked to its final performance, such as friction coefficient [1], wear resistance [2], self-cleaning ability [3], biocompatibility [4], optical response [5], touch perception, overall esthetic aspect, and even flavor [6]. Therefore, it also plays a determinant role in material selection in engineering design, especially in the field of micro- and nano-system development, in which the effects of topography on the incorporation of advanced properties are even more remarkable.

Normally a device surface topography is a consequence of its material's natural state or the result of machining processes, chemical attacks, or post-processes used for the manufacture of a device or product. Several strategies for modifying material topographies and surface properties have taken advantage of conventional surface micro-machining [7], laser ablation [8], micro-molding [9], biomimetic templating [10], physical and chemical vapor deposition processes [11], sol-gel procedures [12], and molecular self-assembly [13]. All these processes require enormous hands-on expertise and final result depends on several control parameters, whose interdependencies are normally complex to understand, characterize, model, and master [14]. As can be seen from the previously cited documents, top-down and bottom-up approaches for 
controlling surface properties co-exist and in many cases complement each other [15], the former being more focused on mass-production (as it derives from the micro-electronic industry), the latter providing remarkable geometrical versatility. Combinations of top-down and bottom-up approaches are frequent and have usually focused on manufacturing the larger micro-metric features by means of top-down processes (micro-machining, etching...) and the smaller nano-metric details by using bottom-up techniques (CVD, PVD, sol-gel...).

Advances in computer-aided design and in additive manufacturing (AM) technologies are opening new horizons for controlling surface topography, of materials and devices, from the design stage and in a very direct, rapid, and easy way. Even though conventional computer-aided design is only capable of handling Euclidean geometries and mainly rely on simple operations (sketch-based operations, extrusions, pads, holes, circular grooves...) for obtaining "soft" solids and surfaces, recent approaches relying on the use of matrix-based programming have already proved to be useful for designing rough surfaces and textured objects adequately described by fractal geometries $[16,17]$. In parallel, the continued progress on additive manufacturing technologies (also called "solid free-form fabrication" due to the complex geometries attainable), especially during the last decade, has increased the range of materials capable of being additively processed and greatly promoted their precision, even down to nano-metric features, with implications in the development of advanced materials, metamaterials, and devices based on them [18, 19].

In spite of the aforementioned potentials, the polymers used in lithography-based AMT, are frequently of limited biocompatibility or inadequate for biomedical applications, what limits enormously the span of final applications. For instance, common thermoplastics used for the mass production of medical devices, including poly(methyl methacrylate) (PMMA) or polycarbonate (PC), cannot be processed using conventional additive manufacturing technologies, and multi-stage processes are required for linking free-form fabrication with mass production [20]. Recent research has also achieved groundbreaking improvements in the bio-compatibility of AM materials $[21,22]$ and dramatically helped to increase the manufacture speed and the attainable precision of these technologies [23]. On the other hand, additive manufacturing technologies aimed at the production of final parts for demanding applications, normally based on layer-by-layer melting or sintering ceramic or metallic powders, do not provide the required precision for obtaining controlled micro-textures and for enabling related advanced functionalities.

Towards the desired degree of precision and performance, LCM technology is a good alternative in terms of detail and attainable aspect ratios. In short, LCM is based on the layer-by-layer selective curing of a photosensitive resin which contains homogeneously dispersed ceramic particles. The photopolymer acts as binder between the ceramic particles and makes the precise shaping of the part possible. The shaped form is produced as a green body that has to be further processed to obtain a part with higher relative density (99.4 \% T.D.). These post-processing steps include the debinding, i.e., the thermal decomposition of the binder and the subsequent sintering into a compact ceramic part. This technology was developed at TU Wien [24-26] and is currently industrially developed by Lithoz GmbH.

In this study, we present the development of two different micro-textured biodevices: a textured cell culture platform and a textured micro-device for studying cell motility. Results show a valuable alternative for precisely defining and controlling the surface topography of ceramic parts from the design stage and for obtaining high-aspect-ratio micro-metric details in ceramic components with complex geometries. Final parts can be directly used for cell culture purposes, due to the adequate properties of the ceramic materials used, without requiring post-processes such as CVD-PVD depositions for promoting biocompatibility or additional replications steps using biomaterials. The strength, toughness, and hardness of the additively obtained ceramic parts make them even candidates for the manufacture of mold inserts for mass production. The following section explains the methods and materials used, before paying attention to the main results obtained, proposing some future directions and detailing our concluding remarks.
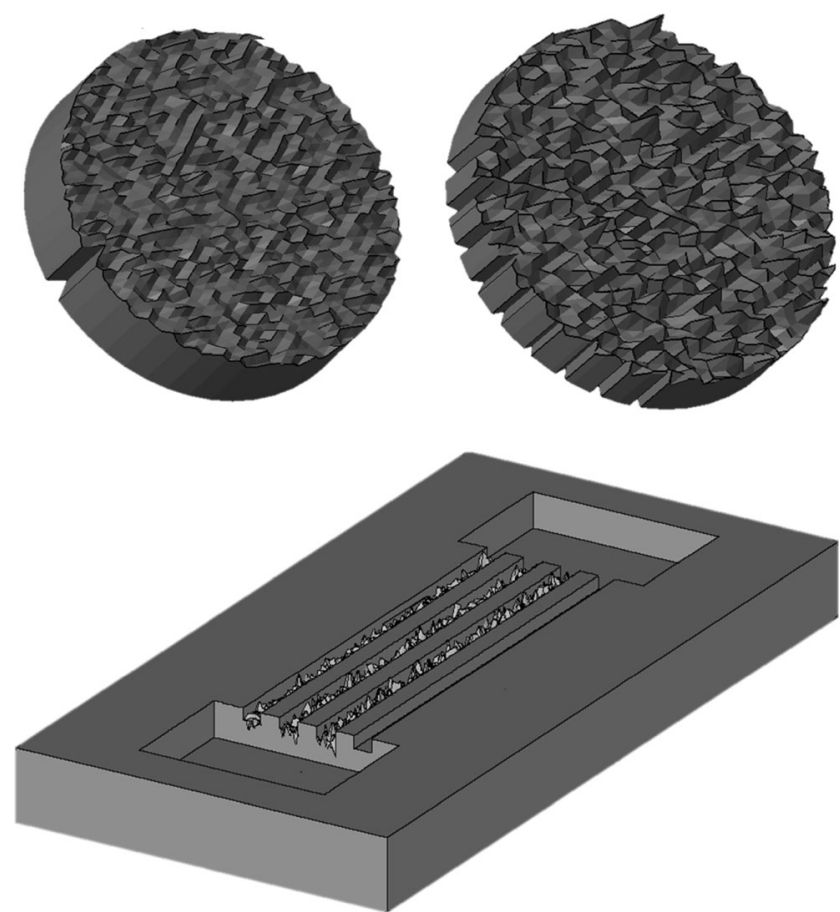

Fig. 1 Computer-aided designs of micro-textured micro-systems. Upper images: Micro-textured cell culture platforms with controlled surface topographies. Lower image: Biodevice for studying cell motility along different micro-textured channels 


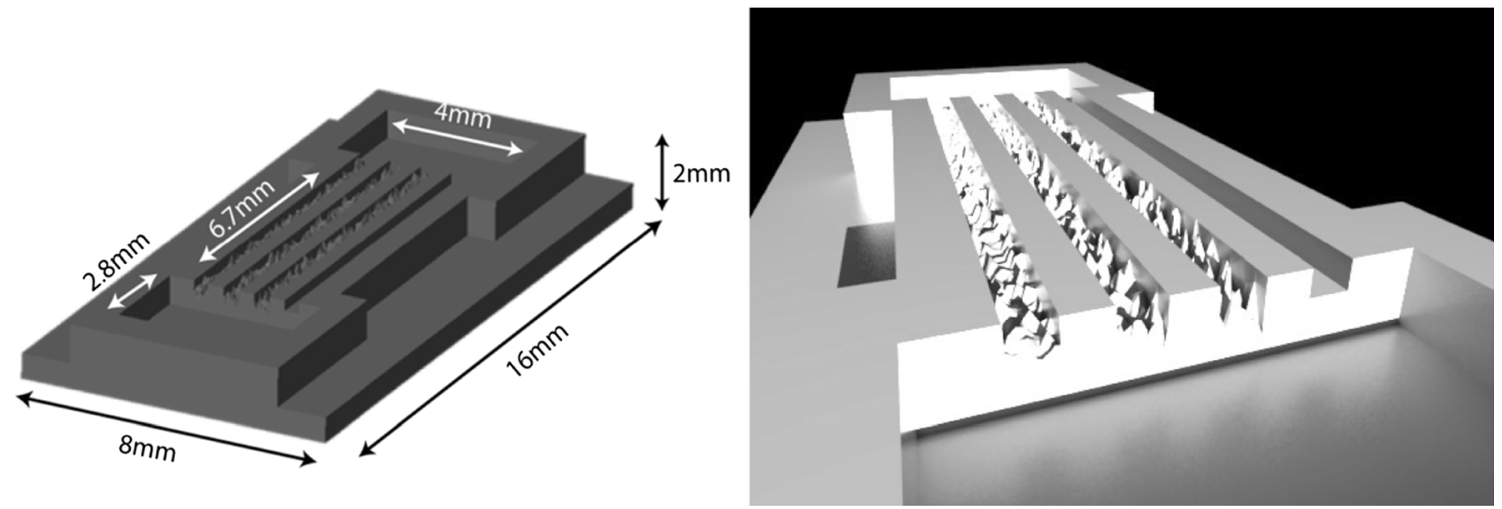

Fig. 2 Design adaptation for enhanced manufacture: Elimination of mass from outer walls to avoid over-exposure and minimize material contraction (left). Rendered and shaded image for improved visualization of the micro-textured channels (right)

\section{Materials and methods}

\subsection{Design process}

The first application example is a set of micro-textured fractal surface-based cell culture platforms, with an improved level of detail, for more adequate interactions at a cellular level than previous preliminary prototypes [27]. The upper images of Fig. 1 show a couple of micro-textured platforms with different roughness levels. These circular platforms are $10 \mathrm{~mm}$ in diameter. In both cases, the upper surfaces include a fractal texture. Typical peak heights lay between 50 and $250 \mu \mathrm{m}$ for the left model and between 350 and $550 \mu \mathrm{m}$ for the right model.

The second application example (shown in the lower image of Fig. 1) corresponds to a micro-system for studying cell motility and addressing the effect of surface texture on cell migration and overall behavior, as previously detailed [28]. The system includes a couple of micro-chambers connected by several micro-channels, for guiding cell movement, each of them with a different texture at its bottom. A typical cell motility experiment should begin with the incorporation of cells to one of the chambers and of growth factors to the other one, so as to promote cell movement from one chamber to another. Channels are $200 \mu \mathrm{m}$ in width and $6 \mathrm{~mm}$ in length. The roughness of the different channels (maximum peak height) reaches values around 5, 5, 100, and $150 \mu \mathrm{m}$, respectively.

Both micro-systems are based on a design procedure firstly described by our team [27] combining different steps, including (a) the generation of fractal textures using fractional Brownian surface models with the help of Matlab (The Mathworks Inc.), (b) the conversion of the fractal surfaces into stl format for further manipulation with computer-aided design programs, (c) the incorporation of thickness to the fractal surfaces using conventional CAD modeling tools, and (d) the combination, by means of Boolean operations, of the textured zones with other solids previously designed, in order to obtain more complex micro-systems or to adjust final size and external shape. Our preliminary in vitro trials with both microsystems were carried upon rapid prototypes adequately coated with diamond-like carbon, to avoid the toxic effects of the acrylic resin, and upon some rapid copies obtained using PDMS casting, as previously detailed [27, 28], and showed promising results.
Fig. 3 Inside of "CeraFab 7500" and building platform. Digital light processing equipment (LCM-Technology,Lithoz $\mathrm{GmbH}$, Vienna)
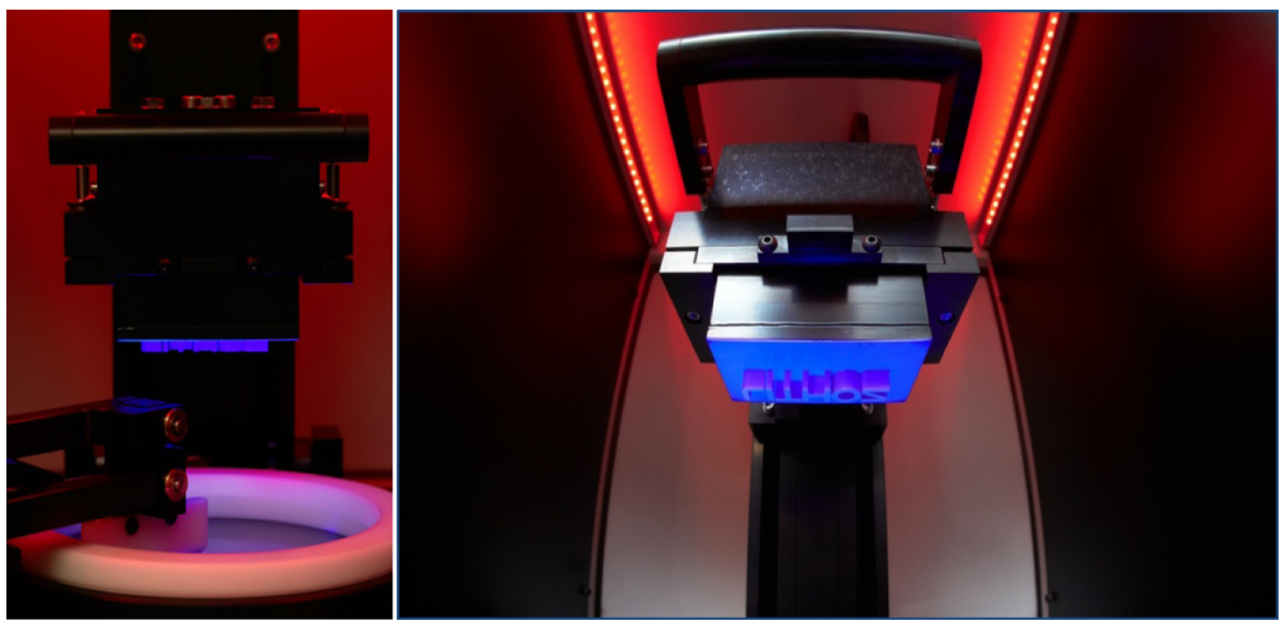

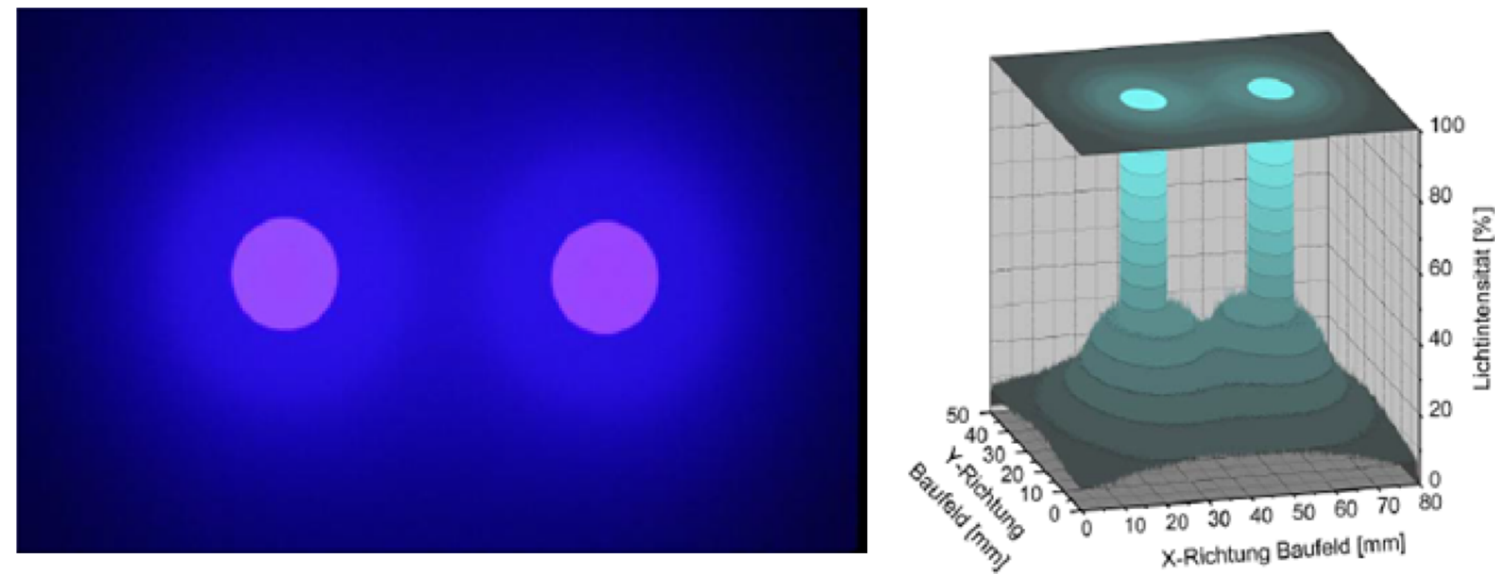

Fig. 4 Overexposure light on the slurry [26]

Figure 2 shows a design adaptation, eliminating the excess of material in the lateral walls of the micro-system by means of Boolean operations, for controlling over-exposure to blue light, which may limit precision, and for minimizing material contraction and related stresses during sintering, as further discussed in the manufacturing section.

\subsection{Additive manufacture of master prototypes or green parts}

Once the design is prepared and optimized for 3D printing, manufacture is accomplished by means of lithography-based ceramic manufacturing (LCM) [29]. The master models or green parts are manufactured, with previously prepared alumina slurries, by digital light processing (DLP) using the CeraFab 7500 machine, Lithoz GmbH (Fig. 3). The slurries are prepared with commercial $\mathrm{Al}_{2} \mathrm{O}_{3}$ powders. These powders are homogeneously dispersed, with the help of a dispersing agent, in a formulation containing reactive monomers and a solvent. In addition, the formulation contains a photoinitiator (typically less than $1 \mathrm{wt} \%$ ). The photoinitiator reacts under an external energy source, in this case a LED emitting at $460 \mathrm{~nm}$, which excites the initiator, creating radicals that chemically react with the monomers included in the mixture. The chain reaction forms the desired matrix of (meth)acrylate monomers that bind together the ceramic particles in the shape of the original part. The reaction occurs in a brief lapse of time, while a determinate section of the part is being projected with specific intensity and exposure time parameters. DLP uses dynamic masks, which represent an individual cross section of the part being manufactured. The light engine uses highperformance LEDs as light source and a DMD chip (digital mirror device) as dynamic mask with a resolution of $1920 \times 1080$ pixels and a pixel size of $40 \times 40 \mu \mathrm{m}$ [29]. The fabrication of the part is done in sequential layer-by-layer manner. For each individual layer, fresh slurry is applied on the building envelope via a dosage system and subsequent rotation of the vat. Afterwards, the building platform is lowered into the dispersion to a distance of $25 \mu \mathrm{m}$ to the bottom of the vat, which equals the thickness of an individual layer in the green body. Then, the space-resolved exposure of the slurry is done by the projection of an image corresponding
Fig. 5 a Example of screenshot from mask layer (layers $n^{\circ} 47, n^{\circ}$ 34 , and $\mathrm{n}^{\circ} 39$ ) for the manufacture of a micro-textured cell culture platform. b Screenshots from masks layers $(30 / 33 / 35 / 37 / 42 / 45$ / 49) for the additive manufacture of the micro-device for cell motility

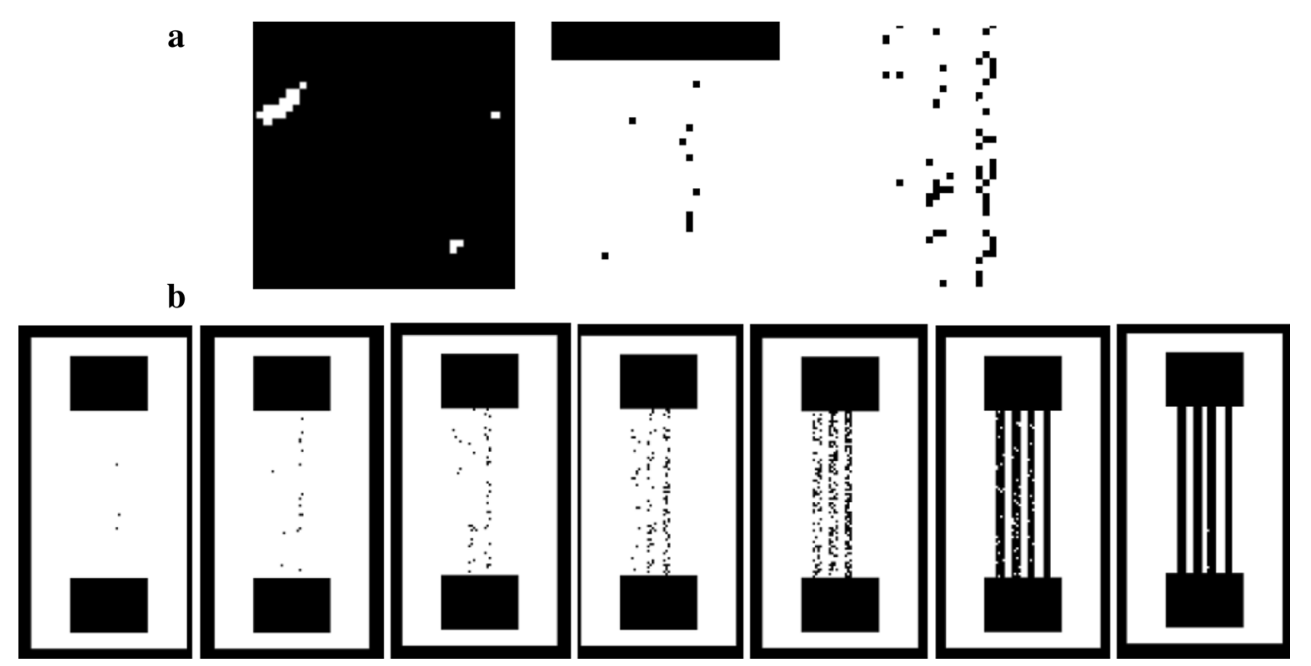



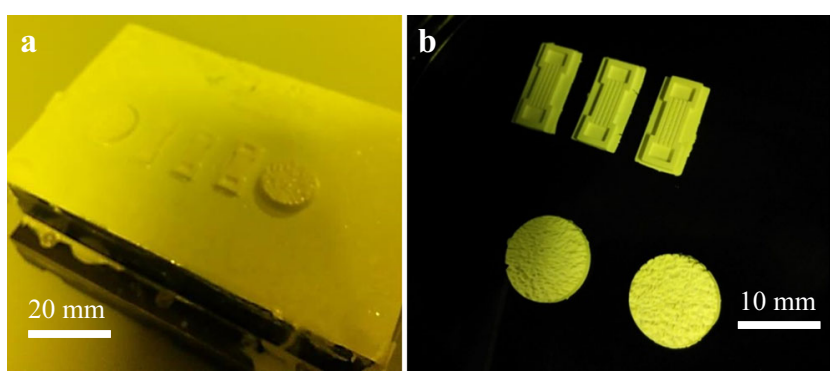

Fig. 6 Green parts in the building platform (a) and cleaned parts after ultrasonic cleaning $(\mathbf{b})$

to the cross section of the current layer. Some images from the mask layers are shown in Fig. 5 by means of example. After printing the parts, they are removed from the building platform. The green bodies are cleaned with solvent for several minutes until the non-polymerized slurry is removed from the cavities of the part.

According to the scheme of Fig. 4, some over-exposure is present, when the light is projected onto the bottom of the batch with the slurry, to form each layer of the part being constructed. This phenomenon, if not adequately taken into account, affects part precision and may prevent the definition of micro-metric details, which in some cases are represented by the DMD as single pixels (see Fig. 5a). Especially in the case of the biodevice for cell motility, very fine peaks must be obtained, so as to precisely control surface roughness from the design stage. With the original CAD design from Fig. 1, overexposure is promoted due to the homogeneous thickness of the part. By means of example, Fig. 5b shows a wide projection zone in several layers aimed at the manufacture of the micro-channels.

To prevent this effect, the re-design shown in Fig. 2 is preferred and provides adequate results. After printing the parts, they are removed from the building platform, which contained a surface photo-reactive resin in order to fix the parts during manufacture. The green bodies are cleaned in an ultrasonic bath with solvent for several minutes until the

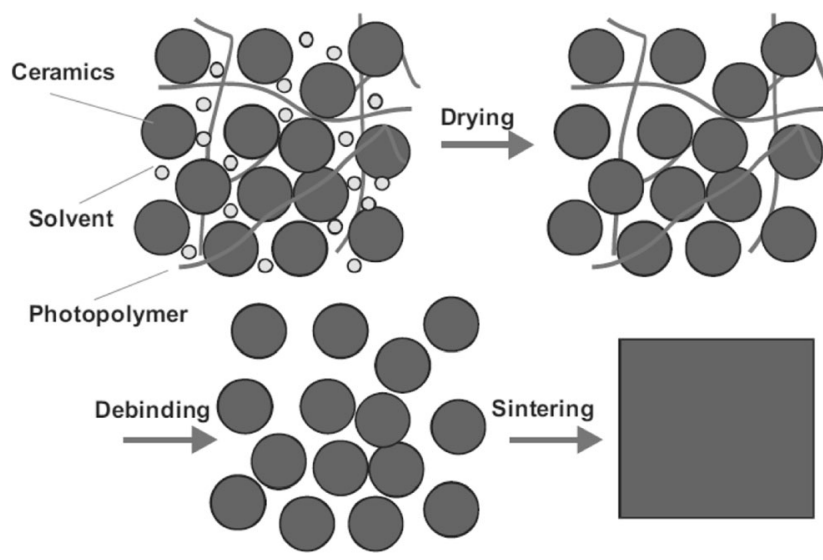

Fig. 7 Steps of thermal treatment from the ceramic green body to the sintered dense ceramic

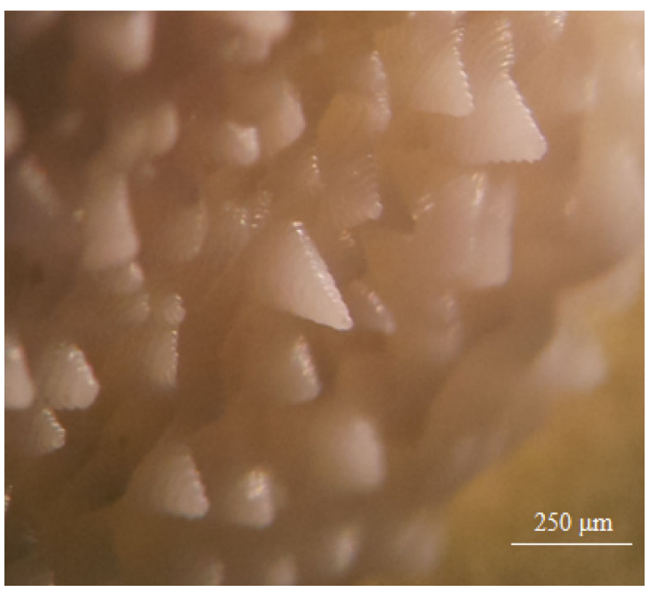

Fig. 8 Sintered parts of micro-textured platforms (dim 2.9) and magnified image of the platform with higher micro-texture for appreciating the spiky feature

non-polymerized slurry is removed from the cavities of the part, as shown in Fig. 6. Actually, ultrasonic cleaning produces cavitation effects, creating bubbles that help to remove the remaining slurry upon the surfaces. Apparently, when looking at the fractal details (Fig. 8), its effect on the final features of the surface is not so critical, although the presence of some bubbles and slight defects (Fig. 12) can be explained by the use of this sort of cleaning, which may sporadically tear some features of the surfaces. Additional research is needed to address the impact, in terms of quality, time, and cost, of alternative cleaning methods.

\subsection{Sintering towards final ceramic prototypes}

The polymeric masters, once the part is free of non-cured slurry, are subjected to a thermal treatment with the aim of obtaining a final ceramic solid part, free of any organic material which could be toxic for the cell development. The binder that makes up a large fraction of the green parts contains substances, which are not chemically compatible with cells. In addition, as the ceramic particles are separated, green parts have lower density and mechanical properties than compact alumina. The elimination of the organic components for achieving final composition and properties is carried out as described in previous research [25]. In short, the thermal variation is controlled inside a high-temperature chamber furnace from 30 up to $400^{\circ} \mathrm{C}$. Firstly, the solvent included in the slurry is evaporated.

Subsequently, a slow temperature variation is provided, so as to get an adequate decomposition of the binder, without causing internal stresses due to the formation of gas at high temperature. In a second step, the $\mathrm{Al}_{2} \mathrm{O}_{3}$ particles are sintered. The part, already free of any organic component surrounding the ceramic particles, is raised up to $1500{ }^{\circ} \mathrm{C}$, hence achieving sintering and resulting in a final compact part. The whole process is schematized in Fig. 7. 
Fig. 9 Comparative images of the surface topographies of the different cell culture platforms (Dim 2.1: left/Dim 2.9: right). Images taken with Olympus DSX-CB tool

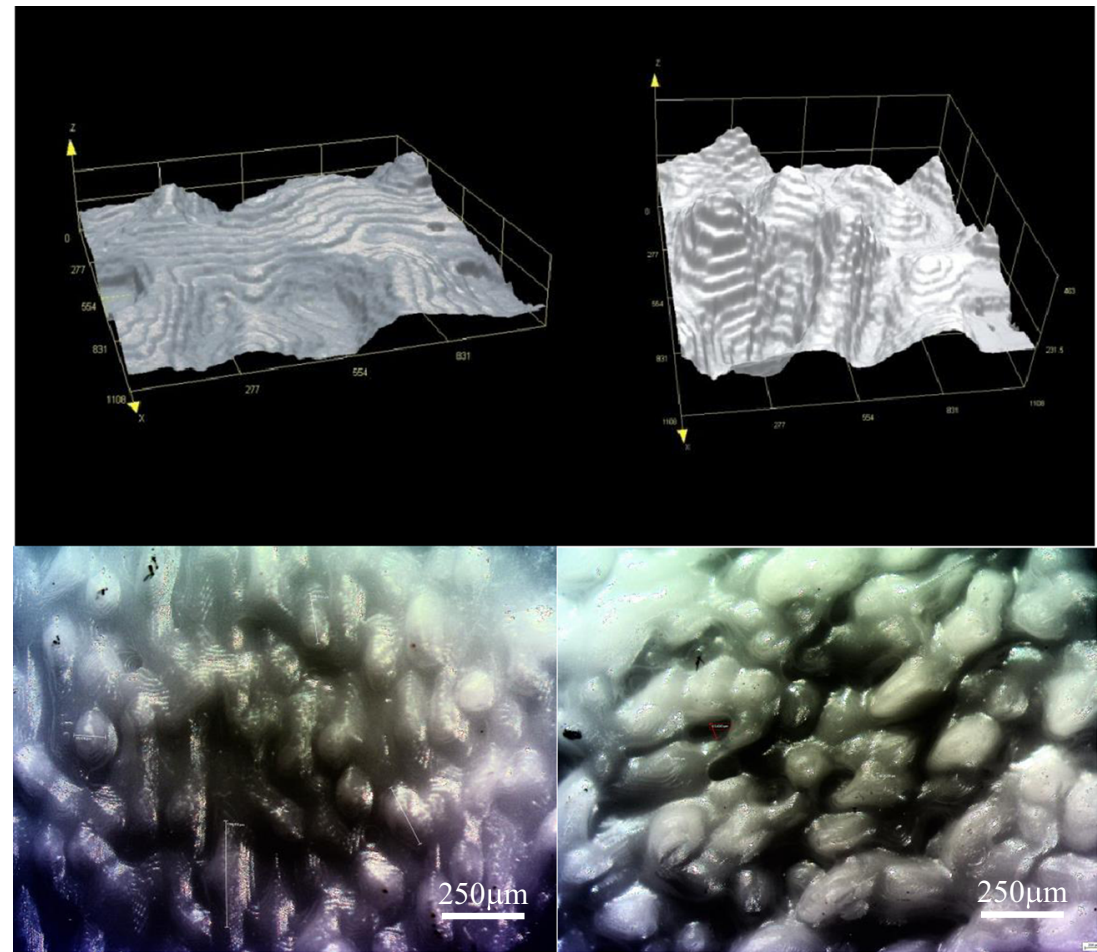

\section{Results}

The manufacture of the micro-textured platforms with different surface topographies shed light about the feasibility of obtaining those complex geometries in ceramic materials using the DLP technology. Figure 8, taken after sintering, includes an image of the micro-textured ceramic platforms magnified with higher micro-texture (2.9 fractal dimension) in order to observe the spiky features in greater detail and the layer which forms the macro-figures. At first glance, the textures adequately reproduce the designs and clear differences can be appreciated between the platform with higher roughness and the platform with lower roughness. When a deeper observation is carried out, 3D representation Olympus DSX$\mathrm{CB}$ tool, the layers conforming the spiky features (and consequence of the additive manufacturing process) can be appreciated. The shape and dimensions are quite similar to the model. Since the chosen layer thickness was $25 \mu \mathrm{m}$, the height they could reach is within the range of $150-500 \mu \mathrm{m}$, as it was expected previously by the fractal model. In the right image of Fig. 9, even 19 layers can be appreciated, corresponding to a $R_{t}$ value of $475 \mu \mathrm{m}$. In the case of the more planar platform, shown in the left image of Fig. 9, around ten layers can be counted, corresponding to a $R_{t}$ value of $250 \mu \mathrm{m}$. These results correspond to the designed values.

After carrying out a final more detailed topographic analysis with Nikon SMZ80C and a digital camera (Imaging Source DFK 72AUCO2) (Figs. 8, 9, 10, 11, and 12), we found some interesting issues, especially in the upper layers. Those differences could be related to a non-homogeneous slurry layer deposition during the building process, also to a not effective polymerization and to the contraction during the sintering process. A direct consequence of the additive manufacture machine precision is the obtaining of master prototypes, in which the last $25 \mu \mathrm{m}$ of the spiky textures are lost, thus leading to somewhat softer surfaces than those from the original
Fig. 10 Photographies of the sintered parts (cell platforms on the left and micro-device for studying cell motility on the right)
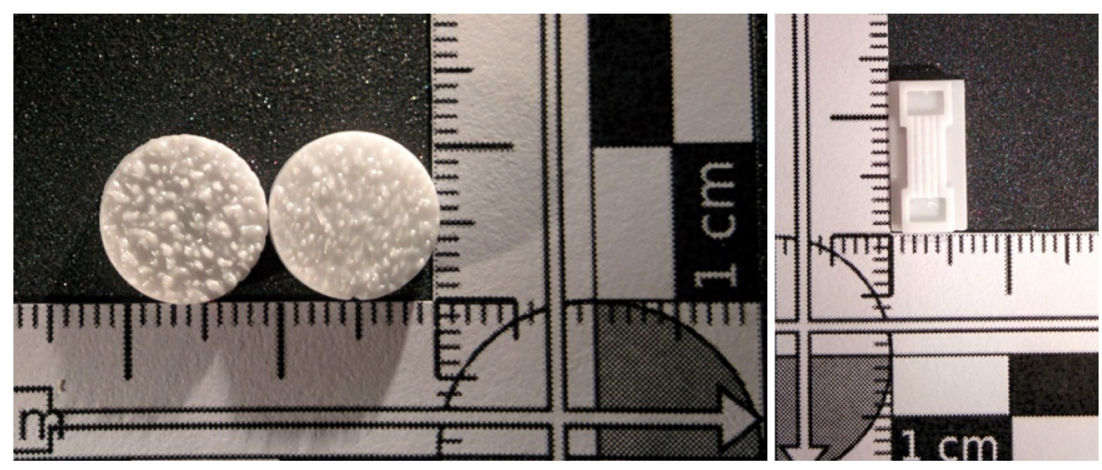


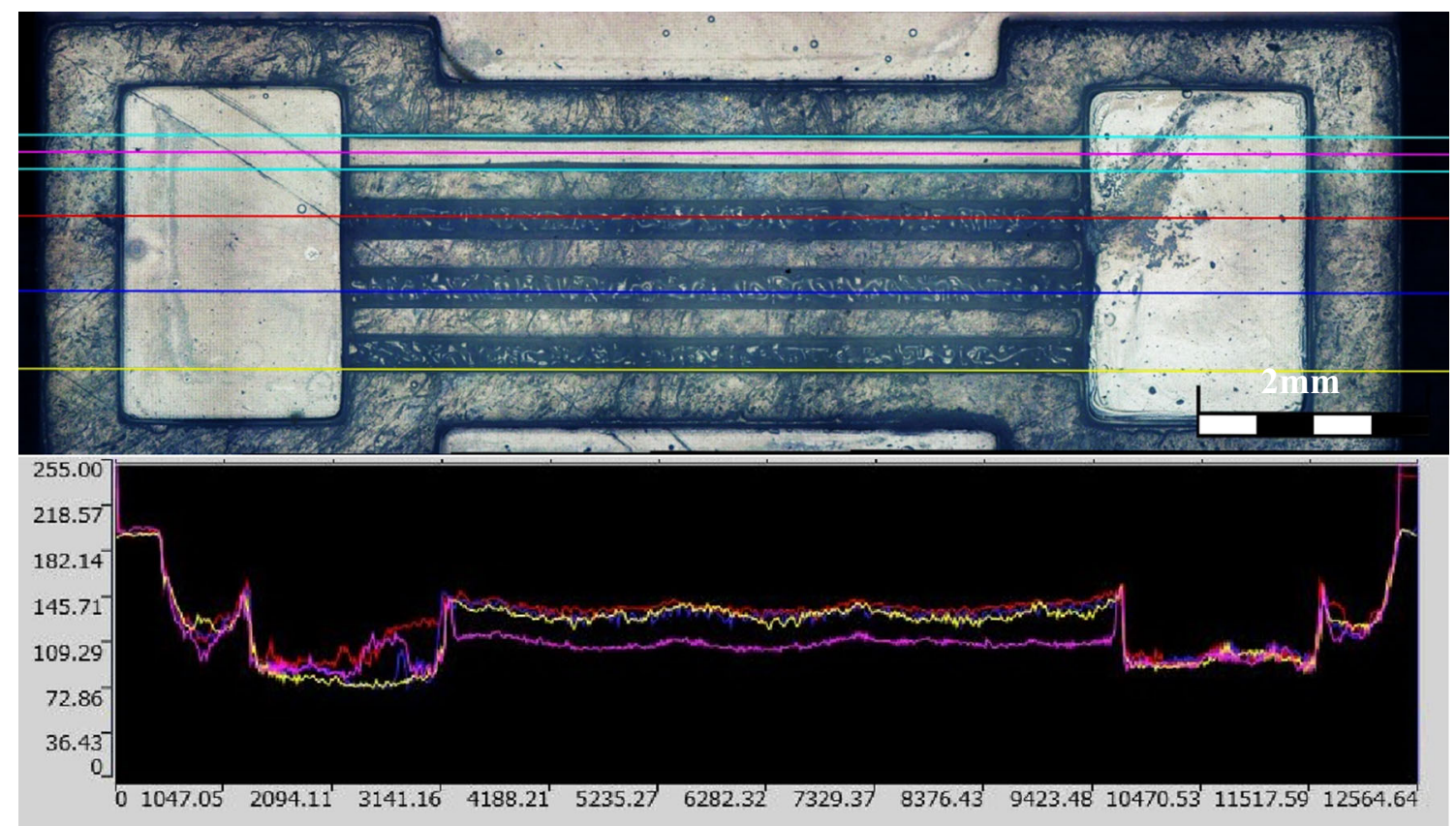

Fig. 11 Profile measurement for each channel with different fractal dimension from up to bottom: 0/2.9/2.5/2.1

CAD files as it can be appreciated in Fig. 9, being the shape even softer for the part of higher dimension, due to more material and layers needed. This effect is shown (Fig. 9) and measured by the size of the "islands" created which are longer in the case of the 2.9 fractal dimension. For most applications

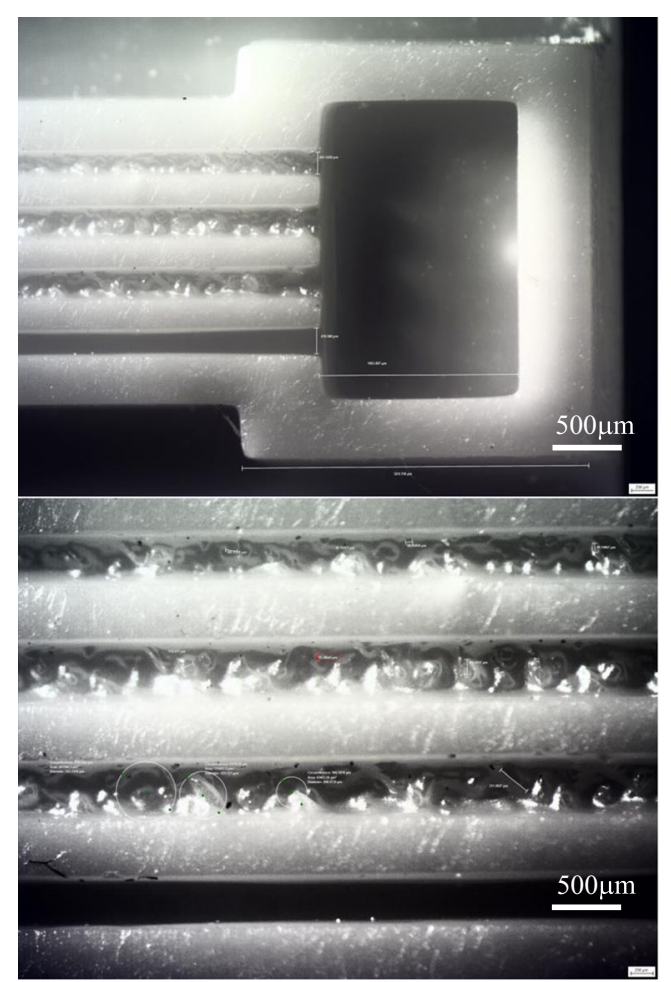

Fig. 12 Detail of micro-channels. a Batch and channel-wide, measured by Olympus software. b Central section of the biodevice of micro-textured surfaces, such softer results may even be positive, as the devices will be a bit softer to the tact and more resistant, as fine needle-like details of $25 \mu \mathrm{m}$ would normally break down under the slightest mechanical request.

The micro-device was also checked by digital microscopy. First results helped us to discover some slight flaws in the micro-chambers, possibly as a result of residual material polymerized inside the batch, which can be appreciated in Fig. 11. After sintering, some contraction is appreciated, but no cracks appear, hence helping us to validate the whole process for the development of micro-textured biomedical microdevices. The dimensional variations can be taken into account from the design stage. Having a look at Fig. 11, the differences can be understood: the original size of the green part has a length of $16 \mathrm{~mm}$, while the sintered part is $13.5 \mathrm{~mm}$ long. These results account for a global volume variation of $19 \%$, which is in accordance with the theoretical results for obtaining a relative density of around $99 \%$. The final lateral dimension (width) of $6.6 \mathrm{~mm}$, when compared with the original dimension of $8 \mathrm{~mm}$, also accounts for around an $18 \%$ of volume variation, which helps to highlight the homogeneity of material contraction. Geometries are in accordance with the original CAD model. The dimension of channels and baths is checked by Imaging Source IC measure software resulting: $203 \mu \mathrm{m}$ wide and $1863 \mathrm{~mm}$ long, respectively (Fig. 12).

In any case, the overall fractal aspect of the surfaces is maintained from the design stage, through the prototyping, to the final sintering step and the whole process is adequate for obtaining micro-textured devices, apt for in vitro trials, and with controlled modifications of surface topography. The last trial over the device was carried out by using the Olympus 
profile-generator tool, which leads to a 3D representation of the object by overlapping a set of images taken with a determinate pitch. As the result of this procedure, the final dimension could be slightly different, for that reason is understood that the heights given by the profiler are not exactly precise. However, the measurements performed help to validate the proposed approach and allow us to check that the fractal peaks manufactured with differences in height and width of less than $19 \%$ when compared with the original CAD.

The process stands out for a manufacturing speed in the range of around 100 layers/hour with a XY plane resolution of $40 \mu \mathrm{m}$ and a layer thickness of $25 \mu \mathrm{m}$, hence being among the most precise and rapid procedures for the manufacture of high-quality ceramic parts, even when compared with very recent developments [30]. In our case, shrinkage is around $19 \%$, well below the $30 \%$ described by using pre-ceramic monomers [30]. The provided images show high-aspect-ratio details with lateral dimensions even lower than $40 \mu \mathrm{m}$ and heights above $500 \mu \mathrm{m}$, which is well beyond an aspect ratio of 10 , which is noteworthy for additively manufactured ceramic parts.

\section{Conclusions}

In this work, we have presented the development of two different micro-textured biodevices which were manufactured by lithography-based AM: a textured cell culture platform and a textured micro-device for studying cell motility. Results show a valuable alternative for precisely defining and controlling the surface topography of ceramic parts from the design stage and for obtaining high-aspect-ratio micrometric details in ceramic components with very complex geometries. The proposed process stands out for the attainable degree of detail, for the high aspect ratios, for the adequacy of final materials for biomedical testing, for the manufacturing speed and for the possibility of obtaining textured micro-systems, which are quite complex to manufacture using conventional micromachining technologies.

Regarding future studies, it will be important to focus on exploring in depth the possible applications of designcontrolled textured surfaces and related devices. We foresee relevant implications for areas including: tribology, due to the potential promotion of adhesion using fractal textures; microfluidics, due to the possibility of controlling the hydrophobicity and hydrophilicity of surfaces by acting on their topography; optics, due to the option of changing surface reflection properties and overall esthetic, and biomedical engineering, for the promotion of biomimetic designs. Currently, the design process, for enabling the introduction of controlled texture gradients and different kinds of texture variations within the surfaces of interest for additional versatility, is being improved.
Acknowledgments We acknowledge Lithoz GmbH for the realization of the prototypes and to the AMT TU Wien department for the support given during the study, as well as the UPM Mechanical department which provided the facilities to measure the parts.

The study has been supported by the European Project "ToMax: Toolless manufacture of complex geometries" funded by the Horizon 2020 Research Program, through the "Factories-of-the-Future" call (H2O2OFoF-02-2014: Manufacturing processes for complex structures and geometries with efficient use of material), under Grant Agreement No. 633192, led by Prof. Dr. Jürgen Stampfl from the Technical University of Vienna.

We are indeed grateful for the detailed review and for reviewers' comments and improvement proposals, which have helped us to incorporate interesting references, to take into account relevant issues and to enhance overall paper quality.

Open Access This article is distributed under the terms of the Creative Commons Attribution 4.0 International License (http:// creativecommons.org/licenses/by/4.0/), which permits unrestricted use, distribution, and reproduction in any medium, provided you give appropriate credit to the original author(s) and the source, provide a link to the Creative Commons license, and indicate if changes were made.

\section{References}

1. Archard J (1974) Surface topography and tribology. Tribol 7(5): 213-220

2. Bushan B, Israelachvili J, Landman U (1995) Nanotribology: friction, wear and lubrication at the atomic scale. Nature 374:607-616

3. Barthlott W, Neinhuis C (1997) Purity of the sacred lotus, or escape from contamination in biological surfaces. Planta 202:1-8

4. Buxboim A, Discher DE (2010) Stem cells feel the difference. Nat Methods 7(9):695-697

5. Berginski M, Hüpkes J, Schulte M, Schöpe G, Stiebig H, Rech B (2007) The effect of front $\mathrm{ZnO}: \mathrm{Al}$ surface texture and optical transparency on efficient light trapping in silicon thin-film solar cells. $\mathrm{J}$ Appl Phys 101:074903

6. Briones V, Aguilera JM, Brown C (2006) The effect of surface topography on color and gloss of chocolate samples. J Food Eng 77(4):776-783

7. Madou MJ. Fundamentals of microfabrication: The Science of miniaturization. CRC Press, 2nd Edition, New York, 2002

8. Chandra P, Lai K, Sunj HJ, Murthy NS, Kohn J (2010) UV laserablated surface textures as potential regulator of cellular response. Biointerphases 5(2):53-59

9. Martin CR, Aksay IA (2005) Microchannel molding: a soft lithography-inspired approach to micrometer-scale patterning. J Mater Res 20(8):1995-2003

10. Pulsifier DP, Lakhtakia A (2011) Background and survey of bioreplication techniques. Bioinspiration Biomimetics 6(3):031001

11. Kwasny W (2009) Predicting properties of PVD and CVD coatings based on fractal quantities describing their surface. J Achiev Mater Manuf Eng 37(2):125-192

12. Jedlicka SS, McKenzie JL, Leavesley SL, Little KM, Webster TJ, Robinson JP, Nivens DE, Rickus JL (2007) Sol-gel derived materials as substrates for neuronal differentiation: effects of surface features and protein conformation. J Mater Chem 16(31):32213230

13. Rahmawan Y, Xu L, Yang S (2013) Self-assembly of nanostructures towards transparent, superhydrophobic surfaces. J Mater Chem A 1(9):2955-2969

14. Gad-el-Hak M (2003) The MEMS Handbook. CRC Press, New York 
15. Naik VM, Mukherjee R, Majumder A, Sharma A. Super functional materials: creation and control of wettability, adhesion and optical effects by meso-texturing of surfaces. Current Trends in Science, Platinum Jubilee Special, 129-148, 2009

16. Mandelbrot B (1982) The fractal geometry of nature. W.H. Freeman, San Francisco

17. Falconer K. Fractal geometry: mathematical foundations and applications. John Wiley \& Sons Ltd., 2003

18. Bückmann T, Stenger N, Kadic M, Kaschke J, Frölich A, Kennerknecht T, Eberl C, Thiel M, Wegener M (2012) Tailored 3D mechanical metamaterials made by dip-in direct-laser-writing optical lithography. Adv Mater 24:2710-2714

19. Röhrig M, Thiel M, Worgull M, Hölscher H (2012) Hierarchical structures: 3D direct laser writing of nano-microstructured hierarchical gecko-mimicking surface. Small 8(19):3009-3015

20. Díaz Lantada A, Piotter V, Plewa K, Barié N, Guttmann M, Wissmann M (2015) Towards mass production of microtextured microdevices: linking rapid prototyping with microinjection molding. Int J Adv Manuf Technol 76:1011-1020

21. Baudis S, Heller C, Liska R, Stampfl J, Bergmeister H, Weigel G (2009) (Meth)acrylate-based photoelastomers as tailored biomaterials for artificial vascular grafts. J Polym Sci A Polym Chem 47(10):2664-2676

22. Baudis S, Steyrer B, Pulka T, Wilhelm H, Weigel G, Bergmeister H, Stampfl J, Liska R (2010) Photopolymerizable elastomers for vascular tissue regeneration. Macromol Symp 296(1):121-126
23. Stampfl J, Baudis S, Heller C, Liska R, Neumeister A, Kling R, Ostendorf A, Spitzbart M (2008) Photopolymers with tunable mechanical properties processed by laser-based high-resolution stereolitoraphy. J Micromech Microeng 18: 125014

24. Gruber H, et al. Rapid-prototyping method and radiationhardenable composition of application thereto. PCT/AT2006/ 000271, WO 2007002965 B1

25. Felzmann R, Gruber S, Mitteramskogler G, Tesavibul P, Boccaccini AR, Liska R, Stampfl J (2012) Lithography-based additive manufacturing of cellular ceramic structures. Adv Eng Mater 14(12):1052-1058

26. Patzer, JF. Generative Fertigung von keramischen Bauteilen für dentale Anwendungen. Dissertation. TU Wien, April 2011

27. Díaz Lantada A, Endrino JL, Mosquera AA, Lafont P (2010) Design and rapid prototyping of DLC coated fractal surfaces for tissue engineering applications. J Phys Conf Ser 252(1):012003

28. Díaz Lantada A. Handbook on advanced design and manufacturing technologies for biomedical devices. Chapter 10. Springer, 2013

29. Schwentenwein M, Homa J (2015) Additive manufacture of dense alumina ceramics. Appl Ceram Technol 12(1):1-7

30. Eckel ZC, Zhou C, Martin JH, Jacobsen AJ, Carter WB, Schaedler TA (2016) Additive manufacturing of polymer-derived ceramics. Science 351(6268):58-62 